\title{
QuantiFERON-TB Gold and the TST are both useful for latent tuberculosis infection screening in autoimmune diseases
}

\author{
F. Bartalesi*, S. Vicidomini\#, D. Golettii, C. Fiorelli\#, G. Fiori' ${ }^{+}$, D. Melchiorre ${ }^{+, \S}$, \\ E. Tortoli $^{f}$, A. Mantella ${ }^{\#}$, M. Benucci**, E. Girardi ${ }^{\dagger}$, M.M. Cerinic ${ }^{+, \S}$ and A. Bartoloni ${ }^{\star * \#}$
}

ABSTRACT: Screening for active tuberculosis (TB) and latent TB infection (LTBI) is mandatory prior to the initiation of tumour necrosis factor- $\alpha$ inhibitor therapy. However, no agreement exists on the best strategy for detecting LTBI in this population. The aim of the present study was to analyse the performance of the tuberculin skin test (TST) and QuantiFERON ${ }_{\mathbb{R}}$-TB Gold in-tube (QFT-GIT) on LTBI detection in subjects with immunomediated inflammatory diseases (IMID).

The TST and QFT-GIT were prospectively performed in 398 consecutive IMID subjects, 310 (78\%) on immunosuppressive therapy and only 16 (4\%) had been bacillus Calmette-Guérin (BCG) vaccinated.

Indeterminate results to QFT-GIT were found in five (1.2\%) subjects. Overall, 74 (19\%) out of 393 subjects were TST-positive and 52 (13\%) were QFT-GIT-positive. Concordance between TST and QFT-GIT results was good (87.7\%): 13 were QFT-GIT-positive/TST-negative and 35 QFT-GITnegative/TST-positive. By multivariate analysis both tests were significantly associated with older age. Only the TST was associated with BCG vaccination and radiological lesions of past TB. Use of immunosuppressive drugs differently modulated QFT-GIT or TST scoring.

Use of the QuantiFERON ${ }_{\mathbb{B}}$-TB Gold in-tube, as a screening tool for latent tuberculosis among immunomediated inflammatory disease subjects, is feasible. Until further data will elucidate discordant tuberculin skin test/QuantiFERON ${ }_{\mathbb{R}}$-TB Gold in-tube results, a strategy of simultaneous tuberculin skin and QuantiFERON ${ }_{\mathbb{B}}$-TB Gold in-tube testing in a low prevalence bacillus CalmetteGuérin vaccinated population, should maximise potentials of latent tuberculosis diagnosis.

KEYWORDS: Interferon- $\gamma$ release assay, latent tuberculosis infection, rheumatic diseases, tuberculin skin test, tumour necrosis factor- $\alpha$ inhibitors

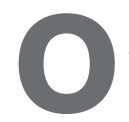
ne-third of the world's population is believed to harbour a latent tuberculosis infection (LTBI) [1], defined as the presence of quiescent mycobacteria. Approximately, $10 \%$ of immunocompetent persons with LTBI will ever develop tuberculosis (TB). In countries with a low incidence of $\mathrm{TB}$, such as Italy, the tracing and targeted treatment of LTBI subjects constitutes a major pillar of TB control [2].

Ageing of the population and the increased use of immunosuppressive treatments represent risk factors for progression to active $\mathrm{TB}$, and highlight the need for additional strategies to maintain and improve TB control [3].

Tumour necrosis factor (TNF)- $\alpha$ inhibitors are approved for the treatment of immunomediated inflammatory diseases (IMID) and provide clinical benefit. However, treatment with TNF- $\alpha$ inhibitors showed an increased risk of serious life-threatening infections, including reactivation of TB infection in patients previously exposed to mycobacteria [4]. Thus, screening for active TB and LTBI has become mandatory prior to the initiation of TNF- $\alpha$ inhibitor therapy $[5,6]$. However, to date, no agreement exists on the best strategy for detecting LTBI in this population, and the tuberculin skin test (TST) remains largely used [7-10].

The TST attempts to measure cell-mediated immunity in the form of a delayed-type hypersensitivity response to purified protein derivative (PPD). However, the TST lacks specificity in populations with high bacillus Calmette-Guérin (BCG) coverage and nontuberculous mycobacteria (NTM) exposure. Moreover, the TST may have a higher probability to have false-negative results in IMID patients than in the general

\section{AFFILIATIONS}

*Infectious and Tropical Diseases Unit, Careggi Hospital, +Internal Medicine Unit, Division of Rheumatology, Careggi Hospital ${ }^{f}$ Tuscany Regional Reference Centre for Mycobacteria, Microbiology and Virology Unit, Careggi Hospital, \# Infectious Diseases Unit, Dept of Critical Care Medicine and Surgery, ${ }^{\S}$ Dept of Internal Medicine, University of Florence,

**Rheumatology Unit, Nuovo San Giovanni di Dio Hospital,

Florence, and

-Clinical Epidermiology Unit, National Institute for Infectious Diseases "Lazzaro Spallanzani", Rome, Italy.

\section{CORRESPONDENCE}

F. Bartalesi,

SOD Malattie Infettive e Tropicali, Azienda Ospedaliero-Universitaria Careggi,

Viale Morgagni 85 ,

50134,

Florence,

Italy.

Fax: 390557949480

E-mail: bartalesif@aou-careggi.

toscana.it

Received:

July 172008

Accepted after revision:

October 292008

STATEMENT OF INTEREST

Statements of interest for

A. Bartoloni, S. Vicidomini, C. Fiorelli,

A. Mantella and D. Goletti can be

found at www.erj.ersjournals.com/

misc/statements.shtml

European Respiratory Journal

Print ISSN 0903-1936

Online ISSN 1399-3003 
population because of the immune disregulation linked to the disease itself or due to immunosuppressive drugs use [11].

Recently, two new assays have been developed to identify LTBI, QuantiFERON®-TB Gold in-tube (QFT-GIT; Cellestis Limited, Carnegie, Australia) and T-SPOT TB (Oxford Immunotec, Abingdon, UK). They measure antigen-specific interferon (IFN)- $\gamma$ secretion by peripheral blood CD4+ T-lymphocytes in response to in vitro stimulation with early secreted antigenic target- 6 and culture filtrate protein 10 [12, 13], which are more specific for the detection of a Mycobacterium tuberculosis infection than PPD, as they are not shared with BCG substrains or with most environmental mycobacteria $[13,14]$.

Several unresolved issues remain on the potential clinical use of IFN- $\gamma$ release assays (IGRAs). One area of controversy is whether they can be used in immunosuppressed patients such as IMID subjects, in addition or as an alternative to the TST. The lack of a gold standard for LTBI diagnosis has hampered the assessment of diagnostic accuracy of IGRAs, and the comparison of these new tests with the TST. Therefore, it has been suggested that tests for LTBI diagnosis can be validated by analysing the association of positive results with risk factors for $\mathrm{TB}$, and different tests can be compared by evaluating differences in their association with risk factors $[15,16]$. The current authors used this approach to compare the performance of QFT-GIT and the TST for the detection of LTBI among IMID subjects coming from a low endemic TB country. Moreover, the impact of different classes of drugs on the response to these tests was analysed.

\section{METHODS}

\section{Study population}

Overall, 398 consecutive subjects were enrolled with rheumatic diseases (rheumatoid arthritis, psoriasic arthritis and ankylosing spondylitis) or other immunomediated chronic diseases, which required the use of biological drugs (infliximab, etanercept, adalimumab and rituximab). The subjects were recruited at two rheumatological outpatient clinics of two hospitals in Florence, Italy (Careggi University Hospital and Nuovo Ospedale San Giovanni di Dio Hospital), from May 2006 to December 2007 and fulfilled published criteria permitting the use of a biological drug [6].

Demographic information, data on BCG vaccination, treatment regimens in the last 3 months and risk factors for LTBI were collected during a standardised patient interview by a researcher who was not aware of the results of the TST or QFT-GIT. Treatments were classified into systemic corticosteroids, conventional disease-modifying antirheumatic drugs (DMARDs; including methotrexate, azathioprine, cyclosporine, leflunomide, cyclophosphamide and hydroxychloroquine) and TNF- $\alpha$ inhibitors (infliximab, etanercept or adalimumab).

All subjects underwent a chest radiograph. The following characteristics were considered to be risk factors for LTBI: birth or residence for $\geqslant 6$ months in a high TB prevalence country (>20 cases per 100,000 inhabitants); history of household TB contact; and healthcare workers who work at facilities where patients with TB are treated.
The following were considered as risk factors for progression to active TB: a medically confirmed history of active TB; chest radiograph findings suggestive of TB history (nodules, fibrotic scars, calcified granulomas or basal pleural thickening); being HIV-positive; body weight of $<10 \%$ of ideal body weight; presence of comorbidity (diabetes mellitus, silicosis, chronic renal failure/haemodialysis, neoplastic or haematological diseases); prolonged therapy with corticosteroids ( $>4$ weeks) or other immunosuppressive therapy; injection drug use; and previous gastrectomy or jejunoileal bypass [17].

\section{QFT and TST}

Blood samples for QFT-GIT were obtained immediately before TST performance. Within $2-6 \mathrm{~h}$ of blood being taken, the samples were incubated at $37^{\circ} \mathrm{C}$. The same biologist, who was unaware of subjects' characteristics and results of the TST, performed all whole blood IFN- $\gamma$ ELISAs according to the manufacturer instructions.

Immediately after blood was drawn, all participants received an injection of 5 units of PPD (Biocine Test-PPD; Chiron, Siena, Italy) by a trained physician. The transverse diameter of induration was measured in millimeters $72 \mathrm{~h}$ later using the ballpen method. TST induration was interpreted relative to risk in accordance with published guidelines [5, 18].

\section{Statistical analysis}

Information from the questionnaires, TST and QFT-GIT results were entered into Microsoft Excel 2003 software. The concordance between QFT-GIT and the TST was assessed by computing the $\kappa$ statistics, with a $\kappa$-value of $>0.75$ representing excellent agreement beyond chance, $0.40-0.75$ representing fair to good agreement beyond chance, and $<0.40$ representing poor agreement beyond chance [19].

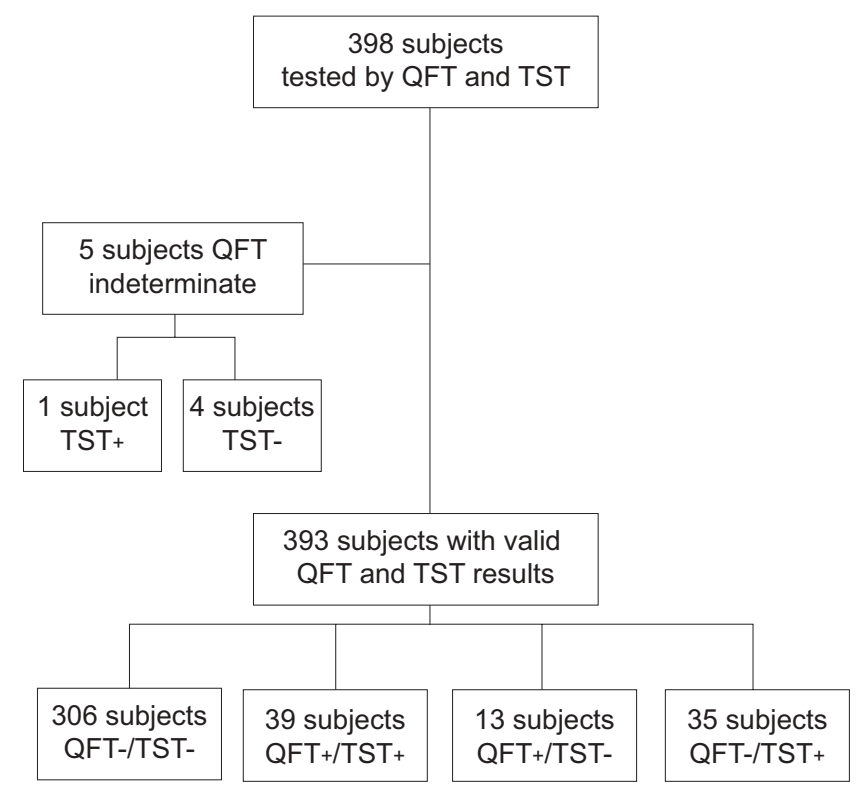

FIGURE 1. Flow diagram of the study population. QFT: QuantiFERONE-TB Gold in-tube (Cellestis Limited, Carnegie, Australia); TST: tuberculin skin testing; +: positive; -: negative. 
TABLE 1 Associations of demographic, epidemiological and clinical characteristics and therapy with QuantiFERON tube (QFT-GIT)- and tuberculin skin test (TST)-positive results in subjects with rheumatic diseases: univariate analysis

\begin{tabular}{|c|c|c|c|c|c|}
\hline & Total & QFT-GIT positive & p-value ${ }^{\#}$ & TST positive & p-value ${ }^{\#}$ \\
\hline Total & $393(100)$ & $52(13.2)$ & & $74(18.8)$ & \\
\hline Male & $137(34.9)$ & $25(18.2)$ & & $32(23.4)$ & \\
\hline Female & $256(65.1)$ & $27(10.5)$ & & $42(16.4)$ & \\
\hline BCG vaccinated & & & 0.1 & & 0.009 \\
\hline Birth/residence in countries at high prevalence of TB & & & 0.7 & & 0.05 \\
\hline Yes & $18(4.6)$ & $2(3.8)$ & & $7(9.5)$ & \\
\hline No & $375(95.4)$ & $50(96.2)$ & & $67(90.5)$ & \\
\hline Age yrs & & & 0.001 & & 0.03 \\
\hline$\leqslant 29$ & $45(11.5)$ & $1(2.2)$ & & $2(4.4)$ & \\
\hline $30-49$ & $129(32.8)$ & $12(9.3)$ & & $25(19.4)$ & \\
\hline No lesions of past TB & $80(20.3)$ & $6(7.5)$ & & $14(17.5)$ & \\
\hline Lesions of past TB & $41(10.5)$ & $9(22.0)$ & & $15(36.5)$ & \\
\hline Other patterns & $272(69.2)$ & $37(13.5)$ & & $45(16.5)$ & \\
\hline Close contact to patients with sputum-positive TB & & & 0.02 & & 0.02 \\
\hline Yes & $7(1.8)$ & $3(5.7)$ & & $4(5.4)$ & \\
\hline No & $386(98.2)$ & 49 (94.3) & & $70(94.6)$ & \\
\hline Diagnosis & & & 0.3 & & 0.4 \\
\hline Rheumatoid arthritis & $201(51.1)$ & $27(13.4)$ & & $35(17.4)$ & \\
\hline Psoriasic arthritis & $94(23.9)$ & $23(24.5)$ & & $16(17)$ & \\
\hline Ankilosing spondilytis & $58(14.8)$ & $5(8.6)$ & & $11(19)$ & \\
\hline Other" & $40(10.2)$ & $4(10)$ & & $5(12.5)$ & \\
\hline \multicolumn{6}{|l|}{ Therapy $^{+}$} \\
\hline TNF- $\alpha$ inhibitors + DMARDs + steroids & $36(9.2)$ & $2(3.8)$ & 0.1 & $2(2.7)$ & 0.009 \\
\hline
\end{tabular}

Data are expressed as n (\%), unless otherwise stated. BCG: bacillus Calmette-Guérin; TB: tuberculosis; DMARD: disease-modifying antirheumatic drugs; TNF- $\alpha$ : tumour necrosis factor- $\alpha$. \#: by Chi-squared test or Fisher's exact test as appropriate. ': includes uveitis, Crohn's disease, juvenile chronic arthritis, undifferentiated spondyloarthropathy ( $n=3$ for each); Behçet's disease, Still's disease, enteritis, temporal arteritis, scleroderma, seronegative oligoarthritis, seronegative spondyloarthritis, ulcerative colitis ( $n=2$ for each); systemic lupus erythematosus, systemic sclerosis, erythema nodosum, Wegener's granulomatosis, polymyalgia syndrome, sarcoidosis, reactive arthritis, psoriasis, myopathy of unclear origin, familial mediterranean fever, sacroileitis and seronegative spondyloarthritis with Castleman's disease $(n=1$ for each). ${ }^{+}$: p-values were derived from comparison of the test results in patients with each single regimen and those of untreated patients. QFT-GIT from Cellestis Limited, Carnegie, Australia.

A standard univariate analysis was performed to analyse the association of the TST and QFT-GIT results to TB risk factors, the type of IMID and the kind of immunosuppressive therapy.

TB risk factors associated with either QFT-GIT or the TST results in the univariate analysis were entered in two separate logistic regression models in which the outcomes were the QFT-GIT or the TST results. In these models, the use of systemic corticosteroids, conventional DMARDs and TNF- $\alpha$ inhibitor therapy were also entered as independent categorical variables.

Finally, to compare the overall diagnostic performance of the two tests, the current authors evaluated whether the association between the number of risk factors for LTBI, selected a priori according to the prognostic relevance [20], and the odds ratio (OR) for testing positive varied according to the test. To this purpose, generalised estimating equations were used to perform 
a logistic regression that accounted for the association between results of QFT-GIT and TST performed on the same subject.

\section{RESULTS}

\section{Studied population}

A total of 398 consecutive subjects with IMID were enrolled (fig. 1). Indeterminate results to QFT-GIT were found in five $(1.2 \%)$ out of 398 subjects. No association was found between risk factors for TB and immune suppression (data not shown) and these samples were excluded for further analysis.

Demographic and clinical features of the 393 remaining subjects are reported in table 1 . The present population was characterised by a high median age ( $54 \mathrm{yrs}$ ), a low prevalence $(4.1 \%)$ of BCG vaccination and a small number $(4.6 \%)$ of subjects coming from high endemic TB countries. None of the enrolled subjects were found to have active TB.

\section{TST and QFT-GIT results}

Concordance between the TST and QFT-GIT

Among the 393 subjects analysed by both the TST and QFTGIT tests, 52 subjects were positive to QFT-GIT and 74 subjects were positive to TST $(p=0.4)$. Among them, $306(77.8 \% ; 95 \%$ confidence interval (CI) 73.4-81.9) subjects were concordant in their negative results and $39(10 \%$; $95 \%$ CI $7.1-13.3)$ subjects in their positive results, resulting in an overall concordance of $87.8 \%$ ( $0.55 ; \mathrm{p}<0.0001 ; 95 \%$ CI $0.44-0.66$ ).

TST-positive/QFT-GIT-negative discordant results were found in $35(8.9 \%)$ out of 393 (95\% CI 6.3-12.2) subjects while TSTnegative/QFT-GIT-positive results were found in $13(3.3 \%)$ out of 393 subjects (95\% CI 1.8-5.6).
Several factors were evaluated to understand their impact on the discordant QFT-GIT/TST data obtained (history of TB exposure, sex, age, history of BCG vaccination, underlying rheumatic disease, etc.). Among them, a history of previous BCG vaccination was associated with discordant QFT-GITnegative/TST-positive results $(p=0.004)$ whereas the use of TNF- $\alpha$ inhibitors was associated with discordant QFT-GITpositive/TST-negative results $(\mathrm{p}=0.03)$.

Risk factors associated with LTBI and type of IMID

By univariate analysis, both positive TST and QFT-GIT were significantly associated with older age and history of close contact with sputum-positive TB patients. However, only TST positivity was associated with BCG vaccination, radiological lesions suggestive of past TB and origin from countries with a high endemic of TB. Only QFT-GIT positivity was significantly associated with males (table 1). These associations were confirmed by multivariate analysis (table 2). No associations between type of IMID and results of the TST and QFT-GIT were found in the univariate analysis (table 1).

\section{Impact of immunosuppressive therapy}

In the univariate analysis, the proportion of positive scoring was lower for both tests for treated patients (with the exception of those treated with DMARDs or DMARDs associated with steroids) compared with the proportion of positive results in patients who were not receiving treatment. A significant association, however, was only found between treatment with steroids and TST results (table 1).

By multivariate analysis, considering the impact of each drug class, the use of DMARDs was not associated with test scoring

TABLE 2 Risk factors associated with QuantiFERON® in-tube (QFT-GIT) and tuberculin skin test (TST) positivity in subjects with rheumatic diseases: multivariate analysis

\begin{tabular}{|c|c|c|c|c|}
\hline & \multicolumn{2}{|c|}{ QFT-GIT positive } & \multicolumn{2}{|c|}{ TST positive } \\
\hline & OR $(95 \% \mathrm{Cl})$ & p-value & OR $(95 \% \mathrm{Cl})$ & p-value \\
\hline BCG vaccinated & NA & NA & $3.8(1.0-13.9)$ & 0.04 \\
\hline \multicolumn{5}{|l|}{ Age yrs } \\
\hline $50-69$ & $6.8(0.8-53.0)$ & 0.06 & $14.7(1.8-117.2)$ & 0.01 \\
\hline$>70$ & $15.9(1.9-132.0)$ & 0.01 & $13.2(1.5-112.7)$ & 0.01 \\
\hline \multicolumn{5}{|l|}{ Chest radiograph patterns } \\
\hline No lesions of past TB & Ref & & Ref & \\
\hline Lesions of past TB & $1.3(0.5-3.2)$ & 0.5 & $3.0(1.3-6.8)$ & 0.005 \\
\hline Other patterns & $0.4(0.1-1.2)$ & 0.1 & $1.6(0.7-3.4)$ & 0.1 \\
\hline Close contacts of patients with sputum-positive TB & $5.3(1-28)$ & 0.04 & $6.5(1.1-36.9)$ & 0.03 \\
\hline Birth/residence in countries at high prevalence of TB & $1.1(0.2-5.8)$ & 0.8 & $3.7(1.1-12.3)$ & 0.03 \\
\hline
\end{tabular}

OR: odds ratio; Cl: confidence interval; BCG: bacillus Calmette-Guérin; TB: tuberculosis; DMARD: disease-modifying antirheumatic drugs; TNF- $\alpha$ : tumour necrosis factor- $\alpha$; NA: not available; Ref: reference. QFT-GIT from Cellestis Limited, Carnegie, Australia. 


\begin{tabular}{|c|c|c|c|c|c|c|}
\hline TABLE 3 & $\begin{array}{l}\text { Associat } \\
\text { and tube }\end{array}$ & $\begin{array}{l}\text { mber of risk fa } \\
\text { (TST) positivity }\end{array}$ & uberculc & ion and Quanti & TB Gold & (QFT-GIT) \\
\hline Number of $r$ & k factors ${ }^{\#}$ & QFT-GIT & & TST po & & p-value \\
\hline & & OR $(95 \% \mathrm{Cl})$ & p-value & OR $(95 \% \mathrm{Cl})$ & p-value & \\
\hline 0 & & 1 & & 1 & & \\
\hline
\end{tabular}

OR: odds ratio; Cl: confidence interval. \#: aged $\geqslant 50 \mathrm{yrs}$, chest radiograph suggestive of a past tuberculosis (TB), close contact with patients with sputum-positive TB, birth/residence in a country with high incidence of TB; ": for the conjoint hypothesis of no difference in the estimates of the two models. QFT-GIT from Cellestis Limited Carnegie, Australia.

while the use of steroids was associated with a lower probability of a QFT-GIT- or TST-positive scoring (OR 0.4, 95\% CI 0.2-09; OR 0.3, 95\% CI 0.2-0.6, respectively; table 2). Moreover, treatment including TNF- $\alpha$ inhibitors significantly decreased the positive outcome of TST (OR 0.3, 95\% CI 0.1-0.6) without affecting QFT-GIT results.

Comparison of the diagnostic performance of TST and QFT-GIT In the absence of a gold standard for LTBI diagnosis, the diagnostic performance of the two tests was compared by evaluating the association of the odds of testing positive with the presence of one or more risk factors for LTBI (aged $>50 \mathrm{yrs}$, chest radiograph suggestive of past $\mathrm{TB}$, close contact with patients with sputum-positive $\mathrm{TB}$ and birth/residence in a country with a high incidence of TB; table 3 ). The results indicate a clear-cut trend for an increase in ORs with an increasing number of risk factors for both tests, and show no significant difference between the two tests. Moreover, the prevalence of LTBI was computed in the present cohort using different combinations of the two tests used (table 4). This analysis approach suggests a better single performance of the TST than QFT-GIT, although the combined used of both tests seems to be the best approach to maximise the sensitivity of the screening.

\section{DISCUSSION}

Immunosuppressed subjects are one of the most important targets for the screening of LTBI because of the high risk of progression to active TB. Despite its widely recognised limitations, the TST remains the standard method for identifying TB infection before TNF- $\alpha$ inhibitor therapy, due to the lack of

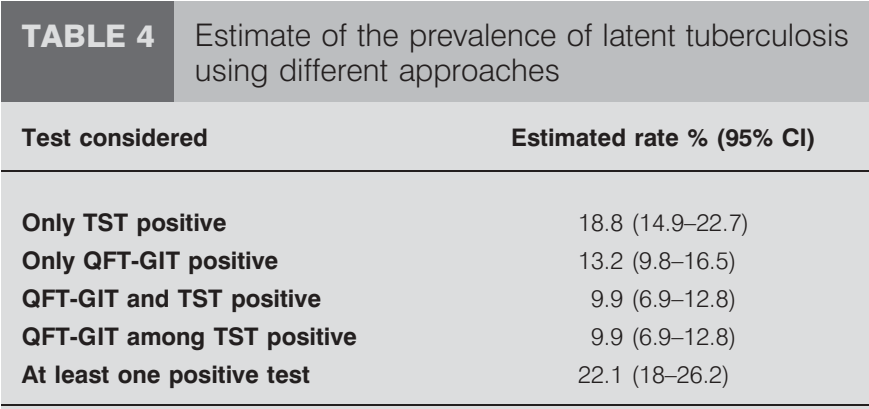

Cl: confidence interval; TST: tuberculin skin test; QFT-GIT: QuantiFERONB-TB Gold in-tube (Cellestis Limited, Carnegie, Australia). sufficient data on IGRA use in this population [7-10]. To date, there are limited data describing performance of IGRA for detecting LTBI in IMID subjects awaiting TNF- $\alpha$ inhibitor therapy. Of them only seven studies reported direct comparison with the TST (table 5) [21-30]. Moreover, the published results were not easily comparable, due to the small number of enrolled subjects, a high rate of BCG vaccination in the studied population, the nonhomogeneous type of underlying diseases considered and the kind of IGRA used (table 5).

To the present authors' knowledge, the current study is the largest that has compared the results of an IGRA to the TST for TB screening in IMID subjects coming from a low endemic TB country (only $4.6 \%$ subjects coming from a high TB prevalence country) and with a low (4.1\%) BCG vaccination rate.

The first relevant finding of the current study was a high positive rate of LTBI for both TST and QFT-GIT testing (18.8 and $13 \%$, respectively). This result was probably due to the high median age of the IMID population ( $55.7 \%>50 \mathrm{yrs})$, who were most likely exposed to the $M$. tuberculosis infection at a young age when Italy was a high TB prevalence country. Therefore, the need for TB screening was confirmed in IMID subjects even in a low TB prevalence country such as Italy.

The present study showed a lower rate $(1.2 \%)$ of indeterminate results at QFT-GIT testing compared with other previously published studies utilising the same test (QFT-GIT) [21, 23, 24, 27], where the rate of indeterminate results ranged between 1.5 and $10.3 \%$ (table 5). The current finding confirms the feasibility of QFT-GIT in the IMID population that have undergone LTBI screening and have been pre-treated with immunomodulating drugs (steroids, DMARDs and TNF- $\alpha$ inhibitors). In total, 79\% of subjects in the present population had been pre-treated.

A fair concordance between QFT-GIT and the TST (87.5\% with $\kappa 0.55 ; \mathrm{p}<0.0001$; 95\% CI 0.43-0.67) was observed, which is higher than reported in previous studies where concordance ranged from $51.7-82.8 \%$ (table 5 ). This is probably due to the lower rate of BCG vaccinated subjects that led to a reduction in the rate of TST-positive/QFT-GIT-negative discordant results compared with the literature, with the exception of the study by PONCE DE LEON et al. [27]. The present interpretation is supported by multivariate analyses showing a significant association between TST-positive/IGRA-negative results and BCG vaccination status $[23,28]$. The current multivariate analysis confirmed this association, although the present 


\begin{tabular}{|c|c|c|c|c|c|c|c|c|c|c|}
\hline \multirow[t]{2}{*}{ Study } & \multirow[t]{2}{*}{ Country } & \multirow{2}{*}{$\begin{array}{l}\text { IGRA } \\
\text { kind }\end{array}$} & \multirow{2}{*}{$\begin{array}{c}\text { Subjects } \\
n\end{array}$} & \multirow{2}{*}{$\begin{array}{c}\text { BCG } \\
\%\end{array}$} & \multirow{2}{*}{$\begin{array}{c}\text { Indeterminate } \\
\%\end{array}$} & \multirow[t]{2}{*}{ к } & \multicolumn{2}{|c|}{ Concordant results } & \multicolumn{2}{|c|}{ Discordant results } \\
\hline & & & & & & & TST+/IGRA+ & TST-/IGRA- & TST-/IGRA+ & TST+/IGRA \\
\hline Cobanoglu [21] & Turkey & QFT-GIT & 68 cases & 100 & 10.3 & 0.14 & $8(13.1)$ & $23(37.7)$ & $1(1.6)$ & $29(47.5)$ \\
\hline MATULIS $^{+}[23]$ & Switzerland & QFT-GIT & 142 & 83 & 6 & 0.16 & $10(7)$ & $60(44.7)$ & $5(3.5)$ & $34(25.4)$ \\
\hline Pratt [24] & UK & QFT-GIT & 101 & 78.5 & 9.9 & & $N A^{\S}$ & $N A^{\S}$ & $N A^{\S}$ & $N A^{\S}$ \\
\hline Kobashi $^{\S}[25]$ & Japan & QFT-G & 252 & 60.3 & 12.6 & 0.29 & $30(13.6)$ & $120(54.5)$ & $6(2.8)$ & $64(29.1)$ \\
\hline DINSER [26] & Germany & $\begin{array}{c}\text { Flow } \\
\text { cytometric } \\
\text { assay }\end{array}$ & 97 & 5.1 & 0 & 0.31 & $6(6.2)$ & 74 (76.3) & $10(10.3)$ & $7(7.2)$ \\
\hline VASSILOUPOLOS [28] & ] Greece & T-SPOT TB & 70 & 40 & 0 & 0.38 & $12(17.1)$ & $39(55.7)$ & $4(5.7)$ & 15 (21.5) \\
\hline \multirow[t]{2}{*}{ BocchiNo [29] } & Italy & QFT-GIT & 69 & 2.8 & 2.8 & 0.57 & $14(20.9)$ & $41(61.2)$ & $8(11.9)$ & $4(6)$ \\
\hline & & T SPOT TB & 69 & 2.8 & 5.8 & 0.48 & $12(18.5)$ & $39(60)$ & $9(13.8)$ & $5(7.7)$ \\
\hline \multirow[t]{2}{*}{ GreenBer [30] } & USA & QFT-G & 61 cases & 27.8 & 11.5 & & NA & NA & NA & NA \\
\hline & & & 42 controls & 23.3 & 2.4 & & & & & \\
\hline Present data & Italy & QFT-GIT & 398 & 4.1 & 1.5 & 0.55 & $39(10)$ & $306(77.8)$ & $13(3.3)$ & $35(8.9)$ \\
\hline
\end{tabular}

BCG: bacillus Calmette-Guérin; +: positive; -: negative; QFT-GIT: QuantiFERON $\mathbb{B}_{\mathbb{B}}$-TB Gold in-tube (culture filtrate protein (CFP)-10, early secreted antigenic target (ESAT)-6 and tuberculosis-7; Cellestis Limited, Carnegie, Australia); ELISPOT: enzyme-linked immunospot; QFT-G: QuantiFERON Carnegie); NA: not available. " ${ }^{*}$ : no direct comparison between the two tests. " ${ }^{":}$ home-made ELISPOT with antigens CFP-10 and ESAT-6. ${ }^{+}$: TST analysed retrospectively. The median time between performing the TST and the QFT-GIT assay was 102 days (range 7-184). ${ }^{\text {s: }}$ nonhomogeneous population including different underlying diseases (74 subjects had malignant diseases, 72 subjects were undergoing immunosuppressive treatment with steroids and/or tumour necrosis factor- $\alpha$ inhibitors, 52 subjects had diabetes mellitus, 50 subjects had chronic renal failure, four subjects had HIV infection). T-SPOT TB manufactured by Oxford Immunotec, Abingdon, UK.

population accounted for only 16 BCG vaccinated subjects. Therefore, these discordant results most likely represent TST associated to a TB infection. The alternative possibility of a past sensitisation to NTM antigens present in the TST and not in the QFT-GIT assay is difficult to ascertain.

Regarding the 13 (3.3\%) TST-negative/QTF-GIT-positive discordant subjects it may be speculated that TST missed these LTBI diagnoses because of the high specificity of QFT-GIT for the $M$. tuberculosis infection. The two main reasons to explain this finding are as follows. 1) A two-step TST was not performed which would have maximised the TST sensitivity, but this strategy would have required four visits instead of two for every subject. 2) All TST-negative/QTF-GIT-positive discordant subjects were undergoing treatment with immunosuppresive drugs and six out of 13 received TNF- $\alpha$ inhibitors, which may explain the negative results of TST, considering the negative impact of steroids and TNF- $\alpha$ inhibitors on M. tuberculosis-specific responses [31] and on the TST performance (table 2). Moreover no association between any LTBI test and kind of IMID were found (table 1). The present results confirm those reported by SELLAM et al. [22] and are consistent with the lack of a relationship with TB infection risk.

Regarding the association of TST and QFT-GIT with risk factors for TB infection, the current data showed a good association for both tests with known risk factors for TB in
Italy, such as older age and a history of close contacts of sputum-positive TB patients. However, the TST seems to be more strictly associated with TB infection, considering that only this test was significantly associated with other known risk factors, such as origin from countries with a high endemic of TB and radiological lesions suggestive of TB history.

Establishing sensitivity and specificity for the $M$. tuberculosis infection is difficult due to the absence of a gold standard for the diagnosis of LTBI. Thus, an approach to index test validation that goes beyond the diagnostic accuracy paradigm is needed for an alternative evaluation process in the absence of a gold standard. In some studies, the association of positive results with risk factors for TB has been analysed, and different tests have been compared by evaluating differences in their association with risk factors $[15,16,20]$. The present authors used this approach to assess the accuracy of the TST and QFTGIT in detecting LTBI, comparing current results to a gradient of risk factors for LTBI selected a priori according to their prognostic relevance. The current findings showed a clear-cut trend for an increase in odds with a growing number of risk factors for LTBI, confirming that both, the TST and QFT-GIT are predictive of LTBI without a difference in the estimation ability between the two tests (table 3 ).

Based on previous studies that showed a reduced performance of the TST in immunosuppressed rheumatoid arthritis subjects 
$[12,27]$, the current authors analysed the impact of different classes of drugs on the response to TST and QFT-GIT tests. By multivariate analysis, steroids were found to significantly affect both QFT-GIT- and TST-positive outcome (OR 0.4 and 0.3 , respectively), while treatment including TNF- $\alpha$ inhibitors significantly decreased only the positive outcome of the TST (OR 0.3). This result disagrees with that reported by MATULIS et al. [23]. However, MATULis et al. [23] did not evaluate the association with the TST results (TST was not performed simultaneously with QFT-GIT). MATULIS et al. [23] explained their result with the TNF- $\alpha$ inhibitors that decrease the activation of CD4+ T-lymphocytes by mycobacterial antigens. However, the present results seem to be more consistent with the crucial role, which TNF- $\alpha$ plays in recruiting macrophages in tuberculin-induced delayed-type hypersensitivity that should justify the reduced response to TST in the current population of whom $24.2 \%$ were already exposed to TNF- $\alpha$ inhibitors at the time of screening [31].

In conclusion, the present study confirms that QFT-GIT as a screening tool for LTBI among IMID subjects awaiting TNF- $\alpha$ inhibitor therapy is feasible, due to the low incidence of indeterminate results and the strict association with risk factors for TB for this test. However, the current findings do not support the recently published UK national guidelines on TB suggesting the use of IGRA as a confirmatory test in TSTpositive subjects [32]. Based on these data in a country with a low prevalence of BCG vaccination, such as Italy, this approach would have had the risk of missing subjects with LTBI. Conversely, the present data suggest the potential use of QFT-GIT as a primary test that may offer the advantage of being less impaired by the treatment with TNF- $\alpha$ inhibitors compared with the TST.

In this context, large-scale cohort studies are needed to estimate risk for progression to active disease in persons who have had positive results to the tuberculin skin test and/or interferon- $\gamma$ release assay, with a particular interest to the risk of disease in persons with discordant reactions. Until further data are available on the implication of discordant tuberculin skin test/QuantiFERON ${ }_{\mathbb{B}}$-TB in-tube results, a strategy of simultaneous tuberculin skin test and QuantiFERON ${ }_{\mathbb{R}}-\mathrm{TB}$ in-tube testing in populations with a low prevalence of bacille Calmette-Guérin vaccination should maximise the sensitivity for latent tuberculosis diagnosis.

\section{REFERENCES}

1 World Health Organization, WHO Report 2008. Global tuberculosis control: surveillance, planning, financing. www.who.int/tb/publications/global_report/2008/pdf/ fullreport.pdf Date last accessed: December 19, 2008.

2 Jensen PA, Lambert LA, Iademarco MF, Ridzon R. Guidelines for preventing the transmission of Mycobacterium tuberculosis in Health-care settings, 2005. MMWR Recomm Rep 2005; 54: 1-141.

3 Horsburgh CR Jr. Priorities for the treatment of latent tuberculosis infection in the United States. $N$ Engl J Med 2004; 350: 2060-2067.

4 Maini R, St Clair EW, Breedveld F, et al. Infliximab (chimeric anti-tumour necrosis factor $\alpha$ monoclonal antibody) versus placebo in rheumatoid arthritis patients receiving concomitant methotrexate: a randomised phase III trial. Lancet 1999; 354: 1932-1939.

5 Gardam MA, Keystone EC, Menzies R, et al. Anti-tumour necrosis factor agents and tuberculosis risk: mechanisms of action and clinical management. Lancet Infect Dis 2003; 3: 148-155.

6 Furst DE, Breedveld FC, Kalden JR, et al. Updated consensus statement on biological agents for the treatment of rheumatic diseases, 2006. Ann Rheum Dis 2006; 65: Suppl. 3, iii2-iii15.

7 British Thoracic Society Standards of Care Committee. BTS recommendations for assessing risk and for managing Mycobacterium tuberculosis infection and disease in patients due to start anti-TNF- $\alpha$ treatment. Thorax 2005; 60: 800-805.

8 Fonseca JE, Lucas $\mathrm{H}$, Canhão $\mathrm{H}$, et al. [Guidelines for the diagnosis and treatment of latent tuberculosis infection and active tuberculosis in patients with inflammatory joint diseases proposed for treatment with tumour necrosis factor $\alpha$ antagonist drugs.] Rev Port Pneumol 2006; 12: 603-613.

9 Mariette X, Salmon D, Group RATIO. French guidelines for diagnosis and treating latent and active tuberculosis in patients with RA treated with TNF blockers. Ann Rheum Dis 2003; 62: 791-792.

10 Beglinger C, Dudler J, Mottet C, et al. Screening for tuberculosis infection before the initiation of an anti-TNF- $\alpha$ therapy. Swiss Med Wkly 2007; 137: 620-622.

11 Ponce de León D, Acevedo-Vásquez E, Sánchez-Torres A, et al. Attenuated response to purified protein derivative in patients with rheumatoid arthritis: study in a population with a high prevalence of tuberculosis. Ann Rheum Dis 2005; 64: 1360-1361.

12 Andersen P, Munk ME, Pollock JM, Doherty TM. Specific immune-based diagnosis of tuberculosis. Lancet 2000; 356: 1099-1104.

13 Pai M, Riley LW, Colford JMJ. Interferon- $\gamma$ assays in the immunodiagnosis of tuberculosis: a systematic review. Lancet Infect Dis 2004; 4: 761-776.

14 Mazurek GH, Jereb J, LoBue P, Iademarco MF, Metchock B, Vernon A. Guidelines for using the QuantiFERON-TB Gold test for detecting Mycobacterium tuberculosis infection, United States. MMWR Recomm Rep 2005; 54: 49-55.

15 Lalvani A, Pathan AA, Durkan H, et al. Enhanced contact tracing and spatial tracking of Mycobacterium tuberculosis infection by enumeration of antigen-specific T cells. Lancet 2001; 357: 2017-2021.

16 Kang YA, Lee HW, Yoon HI, et al. Discrepancy between the tuberculin skin test and the whole-blood interferon gamma assay for the diagnosis of latent tuberculosis infection in an intermediate tuberculosis-burden country. JAMA 2005; 293: 2756-2761.

17 Jasmer RM, Nahid P, Hopewell PC. Clinical practice. Latent tuberculosis infection. N Engl J Med 2002; 347: 1860-1866.

18 American Thoracic Society. Targeted tuberculin testing and treatment of latent tuberculosis infection. Am J Respir Crit Care Med 2000; 161: S221-S247.

19 Fliess JL. The measurement of inter-rate agreement. In: Bradley RA, Levin B, Cho Paik M, eds. Statistical Methods for Rates and Proportions. New York, John Wiley \& Sons Inc, 1981; pp. 212-236. 
20 Kunst H, Khan KS. New tests for the diagnosis of latent tuberculosis infection. Ann Intern Med 2007; 147: 672-674.

21 Cobanoglu N, Ozcelik U, Kalyoncu U, et al. Interferon- $\gamma$ assays for the diagnosis of tuberculosis infection before using tumour necrosis factor $\alpha$ blockers. Int J Tuberc Lung Dis 2007; 11: 1177-1182.

22 Sellam J, Hamdi H, Roy C, et al. Comparison of in vitrospecific blood tests with tuberculin skin test for diagnosis of latent tuberculosis before anti-TNF therapy. Ann Rheum Dis 2007; 66: 1610-1615.

23 Matulis G, Jüni P, Villiger PM, Gadola SD. Detection of latent tuberculosis in immunosuppressed patients with autoimmune diseases: performance of a Mycobacterium tuberculosis antigen specific interferon $\gamma$ assay. Ann Rheum Dis 2008; 67: 84-90.

24 Pratt A, Nicholl K, Kay L. Use of the QuantiFERON-TB Gold test as part of a screening programme in patients with RA under consideration for treatment with anti-TNF $\alpha$ agents: the Newcastle (UK) experience. Rheumatology 2007; 46: 1035-1036.

25 Kobashi Y, Mouri K, Obase Y, Fukuda M, Miyashita N, Oka M. Clinical evaluation of QuantiFERON TB-2G test for immunocompromised patients. Eur Respir J 2007; 30: 945-950.

26 Dinser R, Fousse M, Sester U, et al. Evaluation of latent tuberculosis infection in patients with inflammatory arthropathies before treatment with TNF $\alpha$ blocking drugs using a novel flow-cytometric interferon- $\gamma$ release assay. Rheumatology 2008; 47: 212-218.
27 Ponce de Leon D, Acevedo-Vasquez E, Alvizuri S, et al. Comparison of an interferon- $\gamma$ assay with tuberculin skin testing for detection of tuberculosis (TB) infection in patients with rheumatoid arthritis in a TB-endemic population. J Rheumatol 2008; 35: 776-781.

28 Vassilopoulos D, Stamoulis N, Hadziyannis E, Archimandritis AJ. Usefulness of enzyme-linked immunospot assay (ELISPOT) compared to tuberculin skin testing for latent tuberculosis screening in rheumatic patients scheduled for anti-tumor necrosis factor treatment. I Rheumatol 2008; 35: 1271-1276.

29 Bocchino M, Matarese A, Bellofiore B, et al. Performance of two commercial blood IFN- $\gamma$ release assays for the detection of Mycobacterium tuberculosis infection in pateint candiates for anti-TNF- $\alpha$ treatment. Eur J Clin Microbiol Infect Dis 2008; 27: 907-913.

30 Greenberg JD, Reddy SM, Schloss SG, et al. Comparison of an in vitro tuberculosis inteferon- $\gamma$ assay with delayedtype hypersensitivity testing for detection of latent Mycobacterium tuberculosis: a pilot study in rheumatoid arthritis. J Rheumatol 2008; 35: 770-775.

31 Saliu OY, Sofer C, Stein DS, Schwander SK, Wallis RS. Tumor-necrosis-factor blockers: differential effects on mycobacterial immunity. J Infect Dis 2006; 194: 486-492.

32 National Collaborating Centre for Chronic Conditions. Tuberculosis: clinical diagnosis and management of tuberculosis, and measures for its prevention and control. www.nice.org.uk/CG33\#summary Date last accessed: December 19, 2008. 